\title{
Influence of sample preparation on the multi scale structure of sand-clay mixtures
}

\author{
Kexin Yin ${ }^{1, *}$, Anne-Laure Fauchille ${ }^{1}$, Khaoula Othmani $^{1}$, Giulio Sciarra ${ }^{1}$, Panagiotis Kotronis ${ }^{1}$, Yannick Benoit ${ }^{1}$, \\ Francois Bertrand ${ }^{1}$, and Samuel Branchu ${ }^{1}$ \\ ${ }^{1}$ Université de Nantes, Institut de Recherche en Génie Civil et Mécanique (GeM), Ecole Centrale de Nantes, France
}

\begin{abstract}
This paper focuses on the influence of sample preparation on the multi scale structure of sandclay mixtures. Three different protocols to mix silica and kaolinite were tested in the laboratory to identify the one providing the most homogeneous microstructure. From the macroscopic to the microscopic scales, optical observation, 3D X-ray tomography, 2D scanning electron microscopy (SEM) and 2D environmental scanning electron microscopy (ESEM) were carried out on wet and dry samples. This paper provides a first insight on the mechanisms of sand clay mixing from the $\mathrm{cm}$ to $\mu \mathrm{m}$ scale. Preliminary results demonstrate that the microstructures of the samples prepared by the three procedures have similar macroporosities based on imaging techniques. However, the preparation which consists in mixing the sand firstly, followed by water and clay provides a more homogeneous microstructure with silica grains well-surrounded by an oriented clay layering, probably due to a geometrical effect. Understanding the formation of the oriented clay layering brings microstructural features that will help to better explain the grain displacements and rotations during direct shear tests, the behaviour at the pile sand-clay soil interfaces and to formulate sand clay microstructure models.
\end{abstract}

\section{Introduction}

It is well known that the mechanical behaviour of geotechnical structures as for example offshore marine energy piles and geothermal piles, is mainly governed by the behaviour of interfaces, present at the contact between the soil and the hosting structure and eventually between different soil layers. Interface direct shear tests are usually performed to study the mechanical behaviour of soil-soil and soil-structure interfaces [1-2].

Natural soils are generally classified as sandy or clayey soils based on the fine content classification systems in the laboratory. However, both in-situ and in the laboratory, natural soils are complex and their constituents are characterized by different mechanical behaviours, due to heterogeneous mineralogies, textures and anisotropies. Material's heterogeneities generally modify the sample's behaviour in the laboratory. A nonoptimized control of sample's homogeneity can influence the result variability, which increases the challenge to determine intrinsic parameters.

Despite the number of studies on sand-clay and clayclay mixtures $[3-5,10]$, the effect of sample preparation on sample homogeneity and microstructure, is still not clear enough. In this study, three different protocols to mix sand and clay were tested in the laboratory to identify the one providing the most homogeneous microstructure from the macroscopic to the microscopic scales. Optical observation, 3D X-ray tomography, 2D scanning electron microscopy (SEM) and 2D environmental scanning electron microscopy (ESEM) were carried out on wet and dry samples. The goal is to find the best sample preparation within the three tested, say the one having the most homogeneous structure. This will allow to identify intrinsic properties of soil-soil or soil-structure interfaces.

\section{Materials and methods}

\subsection{Materials}

Fontainebleau sand is used as a reference sand to prepare the sand-clay mixture samples. The parameters of the sand are shown in Table 1. Semi-quantitative SEM analyses show that the sand is composed of $97 \%$ quartz $\left(\mathrm{SiO}_{2}\right)$. The other $3 \%$ are other elements such as $\mathrm{Fe}$ and Al.

An industrial non-swelling kaolinite from Argeco company was chosen to prepare the sand-clay mixtures, in agreement with previous studies [6-9]. It usually contains small grains of minerals other than clay in the fraction below $50 \mu \mathrm{m}$. In this study, the composition and the Atterberg limits were checked (Table 2). The liquid $\left(\mathrm{W}_{\mathrm{L}}\right)$ and plastic $\left(\mathrm{W}_{\mathrm{P}}\right)$ limits of the kaolinite clay we used correspond to the literature [10-11].

\subsection{Sample preparation}

Three different sample protocols are proposed to mix sand, clay and water. In this study, the fractions of sand

\footnotetext{
* Corresponding author: kexin.yin@hotmail.com
} 
and clay are $50 \%$ and $50 \%$ in weight. The samples are mixed with distilled water using a stirring stick in a plastic container during 10 minutes.

The three different mixing procedures are shown in Fig. 1. Sample 1 is mixed by: sand then water then clay; Sample 2 is mixed by: sand then clay then water; Sample 3 is mixed by: clay then water then sand. In sample 1 , clay is added progressively in sand + water, by steps of 5 to $10 \mathrm{~g}$. For sample 2, water is added to the mixture with a spray to ensure the homogeneity of water content. In sample 3, sand is added progressively to clay + water by steps of 5 to $10 \mathrm{~g}$.

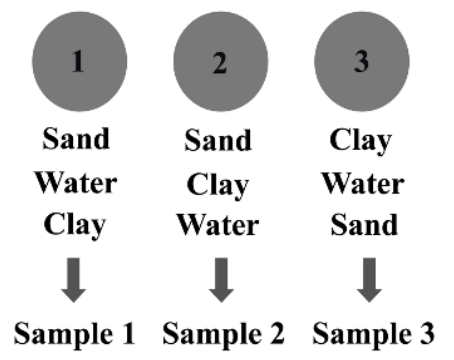

Fig. 1. Scheme of the order of the 3 mixing procedures.

Table 1. Parameters of Fontainebleau sand.

\begin{tabular}{|c|c|c|c|c|}
\hline $\begin{array}{c}\mathrm{d}_{50} \\
(\mathrm{~mm})\end{array}$ & $\begin{array}{c}\mathrm{G}_{\mathrm{s}} \\
\left(\mathrm{g} / \mathrm{cm}^{3}\right)\end{array}$ & $\begin{array}{c}\mathrm{e}_{\max } \\
(-)\end{array}$ & $\begin{array}{c}\mathrm{e}_{\min } \\
(-)\end{array}$ & $\begin{array}{c}\mathrm{Cu}=\mathrm{d}_{60} / \mathrm{d}_{10} \\
(-)\end{array}$ \\
\hline 0.23 & 2.65 & 0.866 & 0.545 & 1.72 \\
\hline
\end{tabular}

Table 2. Parameters of kaolinite clay.

\begin{tabular}{|c|c|c|c|}
\hline $\begin{array}{c}\text { Liquid } \\
\text { limit (\%) }\end{array}$ & $\begin{array}{c}\text { Plastic } \\
\text { limit (\%) }\end{array}$ & $\begin{array}{c}\text { Plasticity } \\
\text { index }\end{array}$ & $\begin{array}{c}\mathrm{G}_{\mathrm{s}} \\
\left(\mathrm{g} / \mathrm{cm}^{3}\right)\end{array}$ \\
\hline 37.3 & 19.0 & 18.3 & 2.63 \\
\hline
\end{tabular}

Distilled water is used to mix sand and clay. Many researchers adopted $1.5 \mathrm{~W}_{\mathrm{L}}$ of the clay as the initial water content to mix sand-clay mixtures [12-15]. Whereas in this paper, the initial water content to mix sand and clay is $1.5 \mathrm{~W}_{\mathrm{L}}$ of the sand-clay mixture $(50 \%$ to $50 \%$ in weight) which measured by Atterberg limits tests (see Table 3). 12 samples were prepared with the three protocols for the later tests.

Table 3. Atterberg limits of sand-clay mixture (50\% to $50 \%$ in weight).

\begin{tabular}{|c|c|c|}
\hline $\begin{array}{c}\text { Liquid } \\
\text { limit (\%) }\end{array}$ & $\begin{array}{c}\text { Plastic } \\
\text { limit (\%) }\end{array}$ & $\begin{array}{c}\text { Plasticity } \\
\text { index }\end{array}$ \\
\hline 22.4 & 13.7 & 8.7 \\
\hline
\end{tabular}

After mixing, the samples were placed in a vacuum device for 1 hour. Then the samples were dried at $105^{\circ} \mathrm{C}$ in an oven for 48 hours. Dry samples were used to make hydric-stable samples for X-ray tomography and SEM analysis.

\subsection{Experimental methods}

Macroscopic observations can get the macroscopic qualitative characteristics of the dry sand-clay mixtures. X-ray tomography was used for the 3D mesoscopic structure of the sand-clay mixture. Scanning electron microscopy (SEM) images and environmental scanning electron microscopy (ESEM) analyses were adopted for the $2 \mathrm{D}$ microstructural approach.

\subsubsection{Optical observation}

Optical macroscopic views of the three different preparations were taken respectively just after drying at $105^{\circ} \mathrm{C}$ for 48 hours (see Fig. 2).

\subsubsection{X-ray tomography}

X-ray tomography scans of the dry samples were acquired with a XRadia Micro XCT-400 device to investigate sample structure at the mesoscopic scale. The tomographic scans were performed for 9 hours each, based on the acquisition of 1800 projections. The resolution of volumes is $12 \mu \mathrm{m}^{3}$ per voxel. The diameter of samples was about $4 \mathrm{~mm}$. One sample of each protocol was put into a thin carbon tube which inner diameter was $4 \mathrm{~mm}$ for scanning. In Fig. 3 there is an example of the detected meso structure.

\subsubsection{Scanning electron microscopy}

Dry specimens of $1 \mathrm{~cm}^{3}$ were carefully cut from each preparation to serve the SEM investigations. Before observation, the specimens were gold-coated to ensure electron interactions on their surface. SEM observations were conducted in a JEOL JSM-6060 LA with a voltage of $20 \mathrm{kV}$ and magnifications between $\times 40$ and $\times 20000$, in secondary and back scattered electron modes.

Observations of microstructure were performed on 2 samples of each mixing protocol, and 8 different areas were observed from each sample.

\subsubsection{Environmental scanning electron microscopy}

The ESEM investigations were conducted in a Zeiss $\mathrm{EVO}^{\circledR} 40$ using a pressure of $946 \mathrm{~Pa}$, at a temperature of $20^{\circ} \mathrm{C}$, a voltage of $20 \mathrm{kV}$, and relative humidities between 88 and $42 \%$. Three humid samples were prepared before the ESEM observations. Back-scattered electron mode was used for a working distance of 5.5 mm. 9 areas of each sample were scanned with standard acquisition parameters. Based on hydric conditions, we have considered the sample's texture under ESEM closer to the natural unsaturated texture than the texture under SEM. 


\section{Results and discussion}

\subsection{Structure at the macroscopic scale}

During mixing, millimetre-size and multi millimetre-size clay aggregates form in sample 2 and sample 3 respectively, contrary to sample 1 (Fig. 2). At the end of mixing, these aggregates are not visible anymore. After drying, minor differences are detected at the macroscopic scale, but there are not any major qualitative differences between the three samples. In sample 1, less macroscopic voids than in samples 2 and 3 are detected. From the point of view of sample homogeneity, one cannot distinguish the differences on the dry samples at this scale.

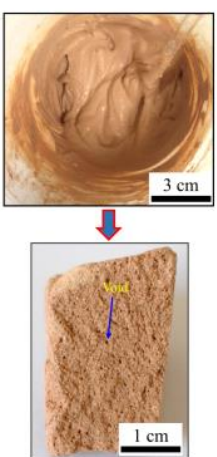

(a)

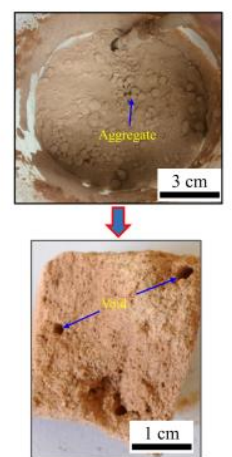

(b)

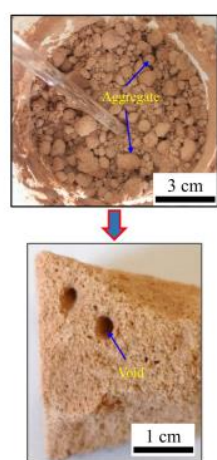

(c)
Fig. 2. Macroscopic views of the 3 samples with different preparations: (a) sample 1; (b) sample 2; (c) sample 3.

\subsection{D structure at the mesoscale}

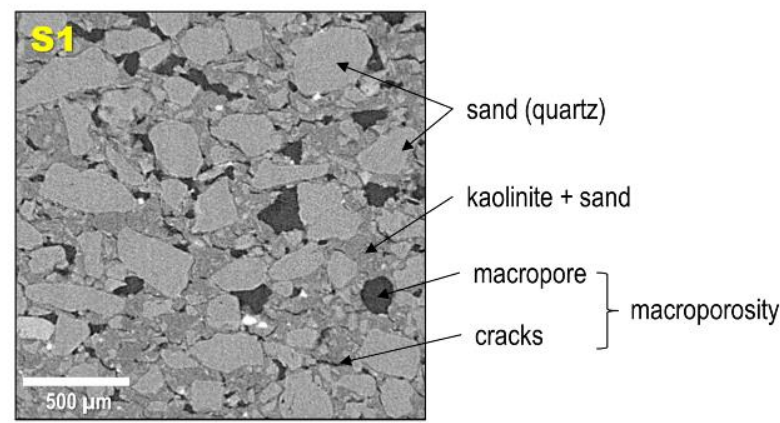

Fig. 3. Example of the microstructure of sample 1 under X-ray tomography.

Sandy grains are easily distinguished from the clay matrix under X-ray tomography due to different densities and grey levels (Fig. 3). Large macropores are visible, due to the heating of samples. The macroporosity is composed of macropores and cracks, in the clay matrix and at the interface between sand and clay, at this scale.

The volume data were segmented in sand, clay matrix and macropores by the ImageJ software to quantitatively investigate the differences in the three preparations. The segmentation threshold of porosity, clay, and sand was performed using the grey level histogram and the non-local mean filter [16-18]. The 3D segmentation was set on volumes of $852 \times 823 \times 1000$ voxels each to avoid any macroporosity artefact from irregular boundaries. The volume fraction of each component was calculated by image analysis (Table 4) and represented in a triangular diagram in Fig. 4.

Table 4. Volume (in voxels) percentage comparison.

\begin{tabular}{|c|c|c|c|}
\hline Sample & Porosity (\%) & Clay (\%) & Sand (\%) \\
\hline 1st & 10.44 & 39.60 & 49.96 \\
\hline 2nd & 9.39 & 39.51 & 51.10 \\
\hline 3rd & 10.22 & 38.56 & 51.22 \\
\hline
\end{tabular}

The macroporosity fractions are $10.44 \%, 9.39 \%$ and $10.22 \%$ in sample 1 , sample 2 and sample 3 . The sand fractions are $39.60 \%, 39.51 \%$ and $38.56 \%$, in addition, the clay fractions are $49.96 \%, 51.10 \%$ and $51.22 \%$ for sample 1, sample 2 and sample 3. Sand fraction is representative of the whole sample. Clay fraction represents less than $50 \%$ in volume due to the macroporosity generated within the clay phase during heating.

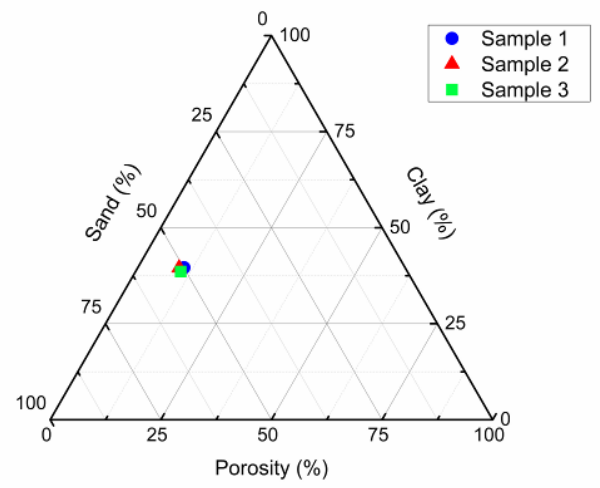

Fig. 4. Triangular diagram of volume comparison in voxels with three components: macroporosity, clay and sand.

The three phase volume fractions are almost overlapping with each other (Fig. 4).

The macroporosity is a little bit higher for sample 1 than samples 2 and 3, but the mean difference between macroporosity fractions is only $0.7 \%$.

\subsection{D microstructure under SEM}

In sample 1 (Fig. 5a), the sandy grains are fully or partly surrounded by a thin clay layer and a few thin cracks are visible in the clay matrix. Clay aggregates partly surround the sandy grains covered by a clay layer. Consequently, sample 1 presents two types of clay media: a thin clay layer which surrounds quartz grains, and a clay matrix between the grains partly structured in aggregates. 
During the preparation of sample 1, sand was firstly mixed with water. During the addition of clays in the sand-water sample, a first part of clay particles went to surround sandy grains probably due to the forced bearing of grains during mixing. The fact that the sandy grains rolled probably facilitated the formation of a thin clay layer around the quartz grains.

In addition, clay aggregates form during or after the deposition of this clay layering, due to chemical attraction of clays in aqueous media.
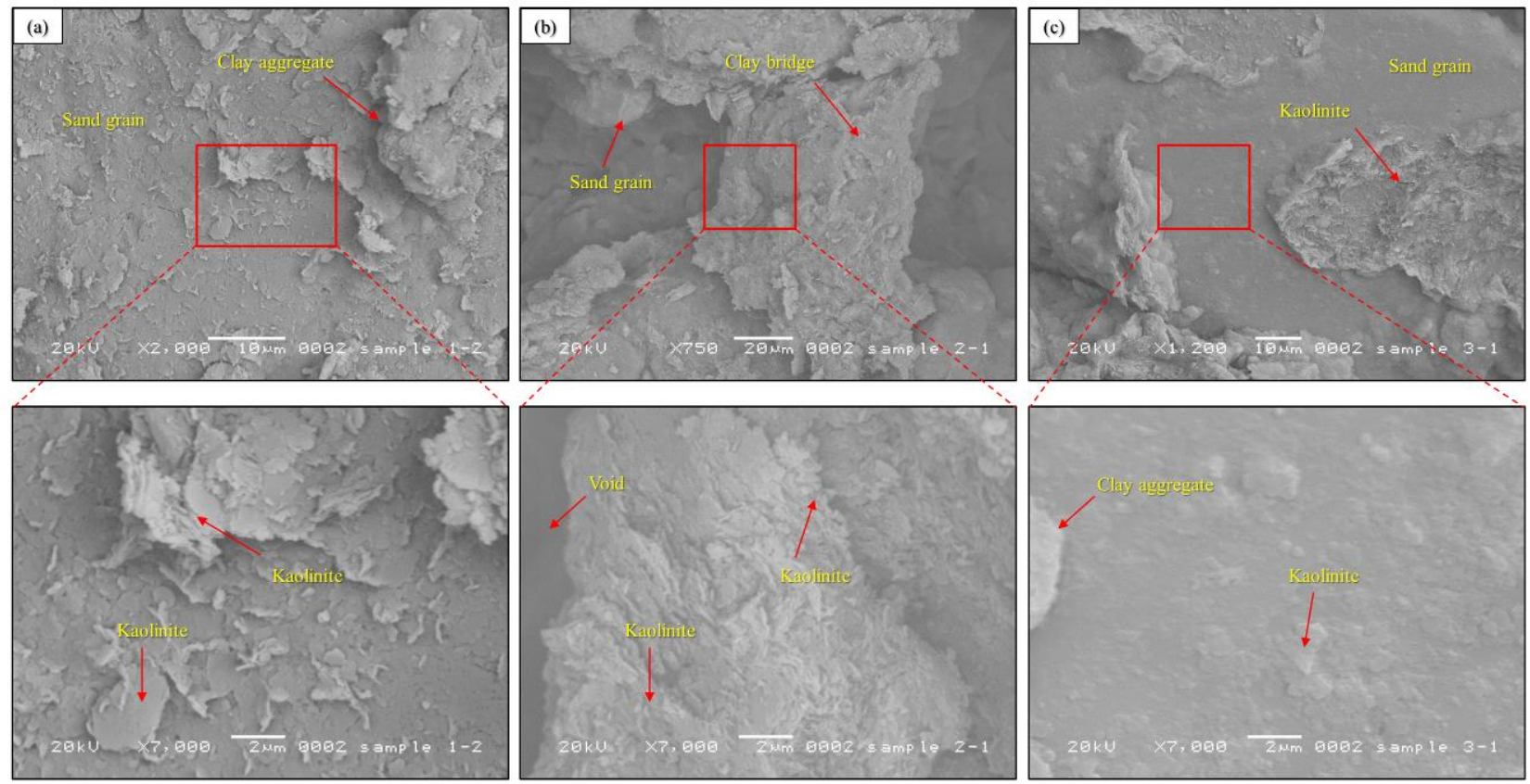

Fig. 5. SEM results of the 3 samples: (a) sample 1; (b) sample 2; (c) sample 3.

In sample 2 (Fig. 5b), the images show a partial clay covering on sand. Some clay particles form clay bridges which connect the sandy grains. Randomly structured kaolinite particles form aggregates, which cover or not the sandy grains. Macropores are observed around the clay bridges. At high magnification, clay particles overlap the sandy grains. Cracks are detected on clay bridges and on clay aggregates, like sample 1. In sample 2 , dry sand was firstly mixed with dry clays, and water was added as the last phase, contrary to sample 1 . In this situation, the bridge formation might be due to the preferential formation of aggregates than clay layering due to the fact that sand and clays were in contact with water at the same time and for same proportions. Moreover, clays could be also removed from sandy grains due to a lowest specific weight during water addition, which could explain the sand areas only partially covered by clays.

In sample 3 (Fig. 5c), sandy grains and clay particles are more differentiated. Clays form a heterogeneous layer around sandy grains with a variable thickness below a few $\mu \mathrm{m}$ corresponding to the size of individual clay crystals. The clay layer is significantly thinner than in the two previous preparations. Both sandy grains wellcovered, partially and poorly covered by clays were observed in this preparation. Clay aggregates and cracks are also present.

In sample 3, water was added to clay firstly, which caused the formation of humid clay aggregates. Then the sand was added, and was consequently mainly mixed to clay aggregates and mud already formed. After adding sand there were not enough individual clay particles left to surround the sand grains completely. At last, the sand grains were covered by clay slurry totally or partially.

According to the 2D micro-scale observations under SEM, the main difference between the 3 sample preparations is the qualitative clay arrangement around the sand grains.

\subsection{D microstructure under ESEM}

By using the environmental scanning electron microscopy (ESEM), 2D high magnification images of the wet samples' microstructures were acquired (Fig.6).

Significant differences of microstructure are not visible for the three samples under ESEM, in comparison with SEM observations (Fig.6).

However, the three samples show an absence of clay particles on sharp sand edges, which are clearly visible in the first, and especially in the second and the third preparations. The absence of clays only on sharp edges might indicate that clays cover preferentially round and smooth surfaces. It shows that clay layering might be driven by a geometrical effect linked to the quartz grain shape. The investigation of this geometrical effect is currently on going.

\section{Conclusions and perspectives}

The multi scale structure of sand clay mixtures, prepared by three different protocols, was investigated by 
different imaging techniques such as optical, 3D X-ray tomography, SEM and ESEM.

The results reveal that the clays within the samples are present as a clay matrix but also as a thin layer around the quartz grains.

The two significant differences between the samples are: 1) the formation of macroscopic clay aggregates during the mixing process for sample 2 and 3 contrary to sample 1, and 2) the presence of a more homogeneous thin clay layer around quartz grains for sample 1 . According to the resolution of X-ray tomography data in which this layer is not visible, this clay layer is probably thinner than a few $\mu \mathrm{m}$. The presence of this clay layering
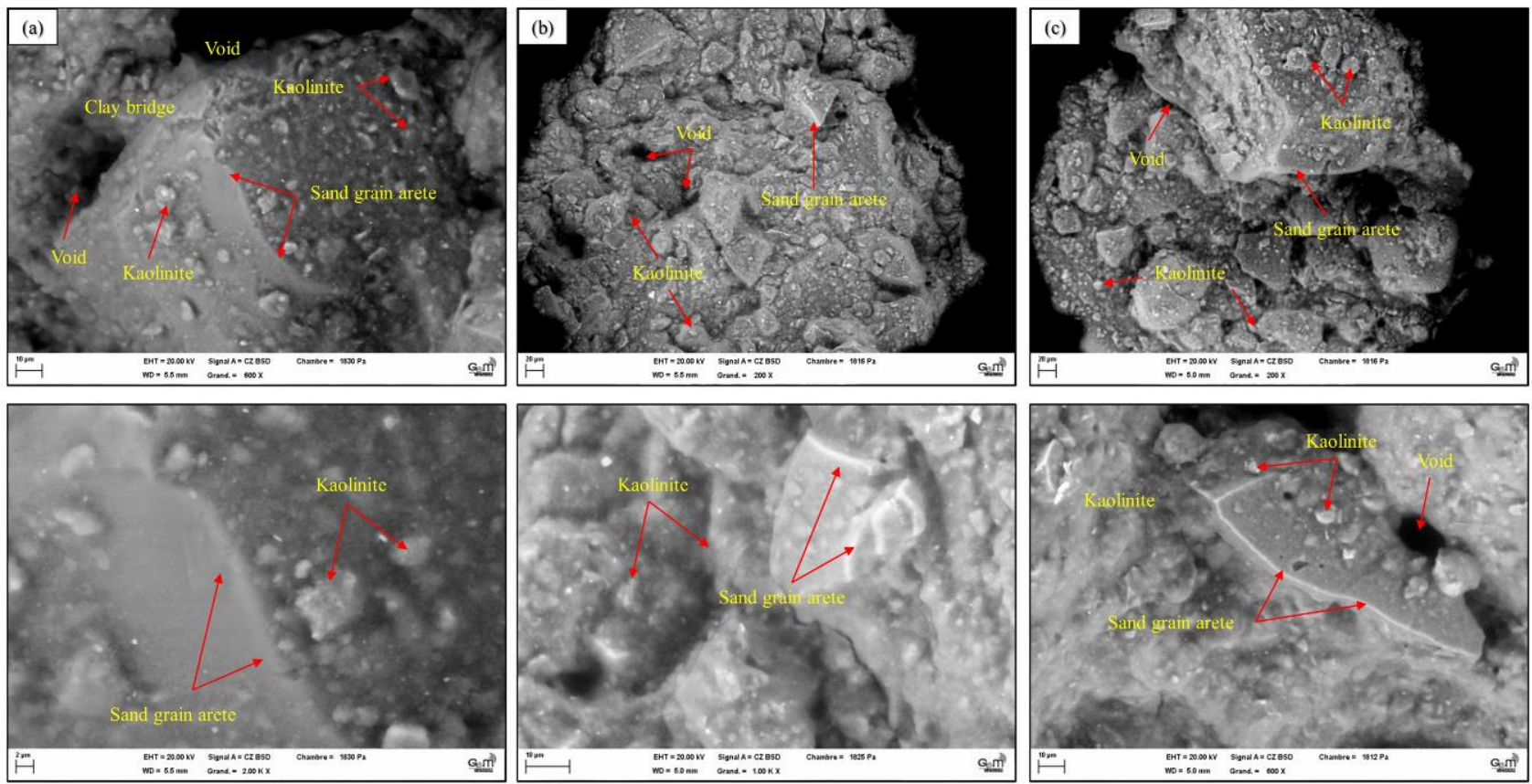

seems to be linked to the shape of quartz grains. No quantitative difference of macroporosity was highlighted with the techniques and the resolutions chosen.

Based on our observations, we preferentially recommend the choice of the first sample preparation for mechanical tests.

In perspective, the role of the different sample preparations on the mechanical behaviour has to be investigated. Microporosity differences will be investigated by mercury intrusion porosimetry (MIP). Robot could also be adopted to mix the samples.

Fig. 6. ESEM results of the 3 samples: (a) sample 1; (b) sample 2; (c) sample 3.

\section{References}

1. G.A. Miller, and T.B. Hamid. Interface direct shear testing of unsaturated soil. Geotechnical Testing Journal. 30, no. 3: 182-191 (2006)

2. G. Mortara, A. Mangiola, and V.N. Ghionna. Cyclic shear stress degradation and post-cyclic behaviour from sand-steel interface direct shear tests. Can Geotech J. 44, no. 7: 739-752 (2007)

3. E.A. Miller, and G.F. Sowers. The Strength Characteristics of Soil-Aggregate Mixtures \& Discussion. Highway Research Board Bulletin, no. 183 (1958)

4. A. Revil, D. Grauls, and O. Brévart. Mechanical Compaction of Sand/Clay Mixtures. J. Geophys. Res. 107, no. B11: 2293 (2002)

5. Z. Lu and B.A. Baudet. Determination of the Transitional Fines Content of Sand-Non Plastic Fines Mixtures. Soils Found. 55, no. 1: 213-219 (2015) 
12. T. Hammad, J-M. Fleureau \& M. Hattab Kaolin/montmorillonite mixtures behaviour on oedometric path and microstructural variations. Eur. J. Environ. Civ. En. 17: 9, 826-840 (2013)

13. M. Hattab, T. Hammad, and J-M. Fleureau. Internal Friction Angle Variation in a Kaolin/Montmorillonite Clay Mix and Microstructural Identification. Geotechnique. 65, no. 1: 1-11 (2015)

14. D. Muir Wood, and G.V. Kumar. Experimental Observations of Behaviour of Heterogeneous Soils. Mech. Cohes. - Frict. Mater. 5, no. 5: 373-398 (2000)

15. G.V. Kumar, and D. Muir Wood. Mechanical Behaviour of Mixtures of Kaolin and Coarse Sand. IUTAM Symposium on Mechanics of Granular and Porous Materials. Solid Mechanics and its Applications, vol 53. Springer (1997)

16. A.L. Fauchille, A.P. van den Eijnden, L. Ma, M. Chandler, K.G. Taylor, K. Madi, P.D. Lee, and E. Rutter. Variability in Spatial Distribution of Mineral Phases in the Lower Bowland Shale, Uk, from the mm-to $\mu \mathrm{m}$-Scale: Quantitative Characterization and Modelling. Mar. Petrol. Geol. 92: 109-127 (2018)

17. J-C. Robinet, P. Sardini, D. Coelho, J-C. Parneix, D. Prêt, S. Sammartino, E. Boller, and S. Altmann. Effects of Mineral Distribution at Mesoscopic Scale on Solute Diffusion in a Clay - Rich Rock: Example of the Callovo - Oxfordian Mudstone (Bure, France). Water Resour. Res. 48, no. 5 (2012)

18. D. Prêt, S. Sammartino, D. Beaufort, M. Fialin, P. Sardini, P. Cosenza, and A. Meunier. A New Method for Quantitative Petrography Based on Image Processing of Chemical Element Maps: Part II. Semi-Quantitative Porosity Maps Superimposed on Mineral Maps. Am. Mineral. 95, no. 10: 13891398 (2010) 